\title{
Carbon Nanofiber Reinforced Aluminum Matrix Composite Fabricated by Combined Process of Spark Plasma Sintering and Hot Extrusion
}

\author{
Hansang Kwon ${ }^{1, *}$, Hiroki Kurita², Marc Leparoux ${ }^{1}$, and Akira Kawasaki \\ ${ }^{1}$ Empa, Swiss Federal Laboratories for Materials Science and Technology, Advanced Materials Processing, \\ Feuerwerkerstrasse 39, CH-3602 Thun, Switzerland \\ ${ }^{2}$ Department of Materials Processing Engineering, Graduate School of Engineering, Tohoku University, \\ 980-8579 Sendai, Japan
}

\begin{abstract}
Spark plasma sintering and hot extrusion processes have been employed for fabricating carbon nanofiber (CNF)-aluminum (Al) matrix bulk materials: The Al powder and the CNFs were mixed in a mixing medium of natural rubber. The CNFs were well dispersed onto the Al particles. After removal of the natural rubber, the Al-CNF mixture powders were highly densified. From the microstructural viewpoint, the composite materials were observed by optical, field-emission scanning electron, and high-resolution transmission electron microscopies. The CNFs were found to be located on every grain boundary and aligned with the extrusion direction of the Al-CNF bulk materials. Some Al carbides $\left(\mathrm{Al}_{4} \mathrm{C}_{3}\right)$ were also observed at the surface of the CNFs. This carbide was created by a reaction between the $\mathrm{Al}$ and the disordered CNF. The CNFs and the formation of $\mathrm{Al}_{4} \mathrm{C}_{3}$ play an important role in the enhancement of the mechanical properties of the Al-CNF bulk material. The CNFs can also be used for engineering reinforcement of other matrix materials such as ceramics, polymers and more complex matrices.
\end{abstract}

Keywords: Metal Matrix Composites, Carbon Nanofiber (CNF), Mechanical Properties, Spark Plasma Sintering (SPS), Hot Extrusion.

\section{INTRODUCTION}

Carbon fiber $(\mathrm{CF})$ reinforced composites have been widely used for industrial materials due to their excellent physical and mechanical properties. ${ }^{1-3}$ Since the discovery of carbon nanotubes (CNT) and carbon nanofibers (CNF), which are extremely small allotropes of carbon unlike conventional CFs, are important for the next generation of materials due to their distinctive properties. ${ }^{4-8}$ Recently, several investigations have attempted to apply CNTs or CNFs for the reinforcement of metal-matrix composites, particularly for aluminum (Al) matrix. Kuzumaki et al. initially fabricated the Al-CNT composites by a hot extrusion method. ${ }^{9}$ Deng et al. ${ }^{10}$ and Laha et al. ${ }^{11-14}$ have also fabricated these using cold isostatic pressing and thermal spraying methods. George et al. demonstrated the strengthening mechanism brought about by CNT in Al matrix composites. ${ }^{15}$ Esawi and Morsi et al. also facilitated the preparation of $\mathrm{CNT}$ reinforced $\mathrm{Al}$ matrix composites by

${ }^{*}$ Author to whom correspondence should be addressed. their unique spark-plasma-extrusion method. ${ }^{16-19}$ However, CNT reinforced Al matrix composites are still far from being reliable and reproducible due to the major problem of unsatisfactory CNT dispersion and difficulty in controlling the interface bonding between the $\mathrm{Al}$ matrix and the CNT. ${ }^{20-24} \mathrm{CNFs}$, which are similar to CNTs, having cylindrical nanostructures with graphene layers arranged as stacked cones, cups or plates ${ }^{25,26}$ should be a suitable candidate for reinforcement material for fabricating of nanocomposites, because CNFs not only have similar characteristics to CNTs but are also easier to handle than CNTs due to their straight shape. Carbon nanofibers with graphene layers wrapped into perfect cylinders are called carbon nanotubes. ${ }^{25}$ Most CNFs are fabricated by a catalytic chemical vapor deposition method. ${ }^{27,28}$

In the present study, we have investigated the potential of $\mathrm{CNF}$ reinforced $\mathrm{Al}$ matrix bulk materials. Natural rubber was used as the mixing medium for homogeneous dispersion of the CNF in the $\mathrm{Al}$ powders then removed by a heat-treatment process. The Al-CNF mixture powders thus obtained were sintered by spark plasma sintering (SPS) 
equipment and then the spark plasma sintered compacts were hot extruded. In particular, pre-sintering of samples is not only helpful for handling compared with the powders but can also be protected from oxidation problems by reduction of the surface area. The mechanical properties of the Al-CNF bulk materials have been investigated based on a microstructural view point.

\section{EXPERIMENTAL DETAILS}

Commercial gas-atomized $\mathrm{Al}$ powder (ECKA Granules Japan Co. Ltd, purity $99.85 \%$, average particle size $15 \mu \mathrm{m}$ ) and vapor grown carbon nanofibers (CNFs) (Showa Denko Co. Ltd, purity over $99 \%$, diameter $100 \mathrm{~nm}, 10 \mu \mathrm{m}$ in length), shown in Figures 1 and 2 were used as the starting materials. At first, a precursor, designed to be a homogeneous dispersion of $\mathrm{CNF}(5 \mathrm{vol} \%)$ in the $\mathrm{Al}$ powders, was prepared using a mixing medium of natural rubber by a NSD method. ${ }^{29-32}$ The precursor was heat-treated at $500{ }^{\circ} \mathrm{C}$ for $2 \mathrm{~h}$ in an argon atmosphere $(1 \mathrm{l} / \mathrm{min})$ to evaporate the natural rubber. The Al-CNF mixture powder thus obtained was sintered in a carbon mold using a spark plasma sintering device (SPS-S515) manufactured by Sumitomo Coal Mining Co. Ltd. The sintering temperature and conditions were $600{ }^{\circ} \mathrm{C}$, holding time of $20 \mathrm{~min}$, heating rate of $40^{\circ} \mathrm{C} / \mathrm{min}$, and pressure of $50 \mathrm{MPa}$.
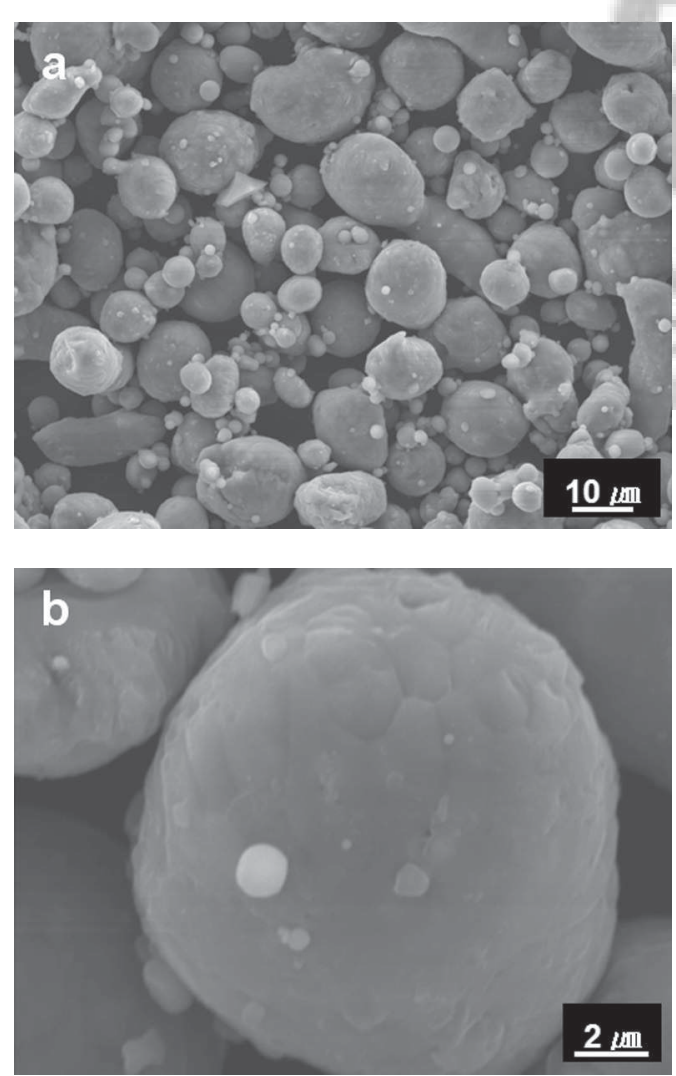

Fig. 1. SEM micrographs showing the shape of the $\mathrm{Al}$ particles employed in this study, (a) low magnification and (b) high magnification.
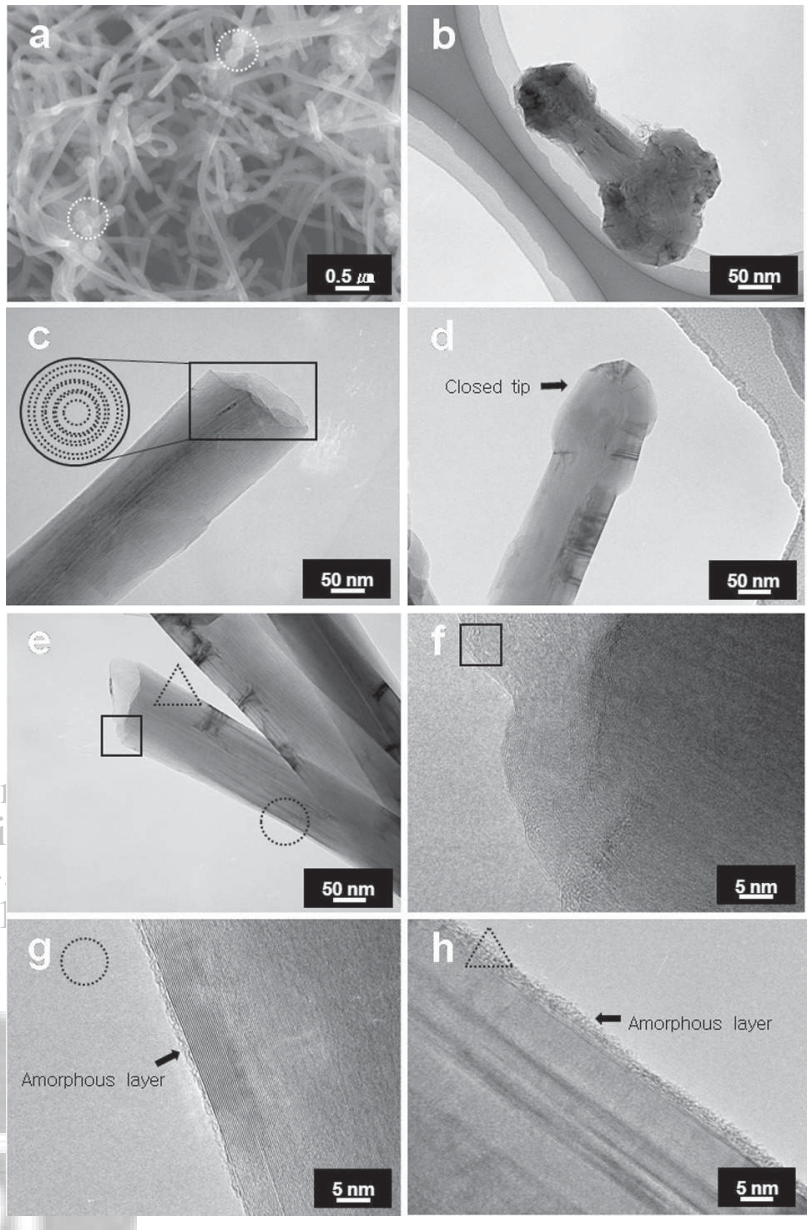

Fig. 2. Micrographs of the raw CNF employed in this study.

The size of the sintered compact cylinder was $\phi 15 \mathrm{~mm}$ and $30 \mathrm{~mm}$ in length. The SPS Al-CNF compact was extruded in a $60^{\circ}$ conical die at $400{ }^{\circ} \mathrm{C}$ with a pressure of 500 kN (UH-500 kN1, Shimadzu Corporation, Japan). The extrusion velocity and the extrusion ratio were fixed at $2 \mathrm{~mm} / \mathrm{min}$ and 20, respectively.

The microstructures of the samples after every step were observed by an optical microscope (PMG-3, Olympus, Japan), field-emission scanning electron microscope (FE-SEM6500, JEOL Co. Ltd, Japan) and high-resolution transmission electron microscope (HR-TEM, Hitachi, Japan) with selected-area diffraction pattern (SADP) measurements. The Raman spectroscopy (SOLAR TII Nanofinder, Tokyo Instruments Co. Ltd, Japan) served to evaluate the disorder of the CNFs. The microhardness of the raw $\mathrm{Al}$ particle and extruded bulks were measured by a nano-indentor (TMZ-512, Akashi Co. Ltd, Japan) using a load of $0.02 \mathrm{~N}$ for five measurements per sample. For evaluation of tensile strength, the extrudates were machined into test pieces $3 \mathrm{~mm}$ in diameter in accordance with ICS 59.100.01. The tensile strength was measured by a universal testing machine (AUTOGRAPH AG-I $50 \mathrm{kN}$, Shimadzu Co. Ltd, Japan). 


\section{RESULTS AND DISCUSSION}

Figure 2 shows FE-SEM and TEM micrographs of the raw CNF. Several tips of the CNFs showed a lump, indicated by white circles in Figure 2(a). These may be formed during the manufacturing process due to contaminants such as carbon black or catalysis materials etc. Most of the raw CNFs have been observed with two kinds of tip shapes, namely open-tip and close-tip, as shown in Figures 2(c and d). In particular, some of the inner walls of open-tipped CNFs contained rugged graphene morphology (see Fig. 2(f)). Some amorphous layers, about $2 \mathrm{~nm}$ thick, were observed on the surface of the CNFs, as shown by the circle in Figure 2(g) and the triangle (h), at high magnification, in Figure 2(e).

Figure 3 shows Raman spectra $^{33,34}$ of the raw CNFs. Even though the pristine CNF included a defect at the open tip and an amorphous layer, the ID/IG ratio has a low value, which means that the CNF is of relatively high quality. ${ }^{33}$

Figure 4 shows FE-SEM micrographs of the Al-CNF mixture powders after removal of the natural rubber. The $\mathrm{CNF}$ was randomly dispersed on to the Al particles. No residual natural rubber was observed in the Al-CNF mixture powders, at least at the FE-SEM level of analysis. From the viewpoint of dispersion, there are two kinds of mixed powders observed, one of which has a small quantity of the CNF on the Al particle and the other a relatively large quantity, as shown in Figures 4(a and b). This phenomenon not only depends on the dispersion method but is also closely related to the volume ratio between the $\mathrm{Al}$ and CNF. No highly agglomerated regions were observed in the Al-CNF mixed powders overall. This indicates that the straight shape of the CNF offered lower potential for mechanical capture, resulting in restrained CNF agglomeration (Fig. 2). In conclusion, we believe that using natural rubber as the mixing medium for homogeneous dispersion of the CNFs on to the Al particles is a useful technique.

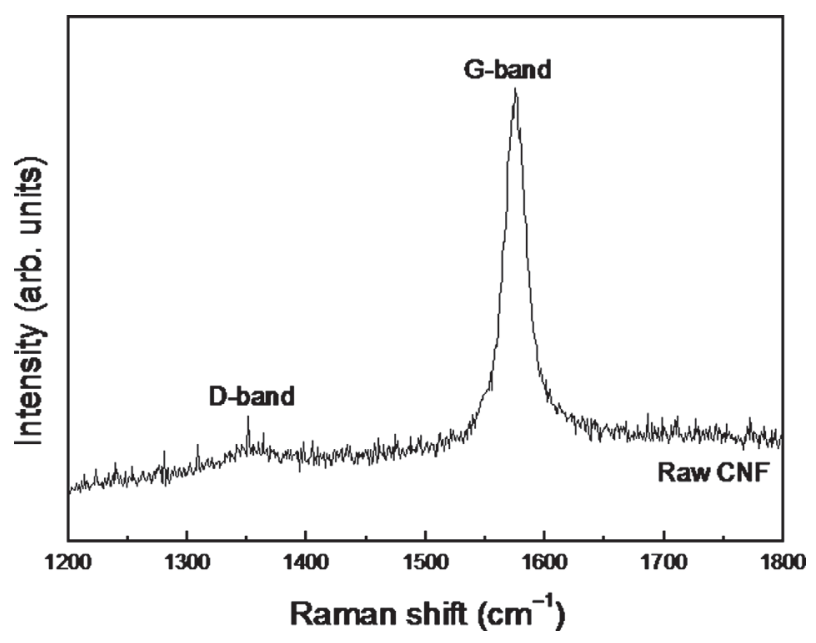

Fig. 3. Raman spectra of the raw CNF.
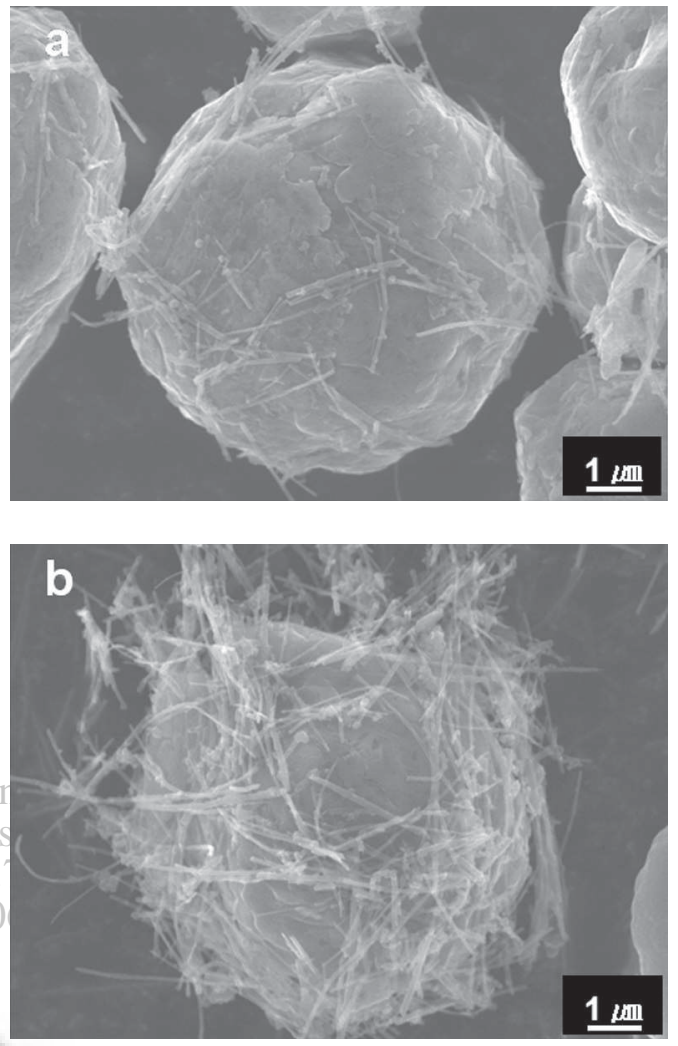

Fig. 4. FE-SEM micrographs of Al-CNF mixture powder. (a) little CNF around the $\mathrm{Al}$ particle and (b) much more $\mathrm{CNF}$ around the $\mathrm{Al}$ particle.

Figure 5 shows micrographs of longitudinal and transverse cross sections of the SPS Al-CNF compact. The CNFs were well dispersed at every boundary and the shape was similar to the starting CNF even after the SPS process, as shown in Figures 5(b and e) and Figure 2. The shape of the $\mathrm{Al}$ particles in the longitudinal cross section were only slightly distorted perpendicular to the direction of the uniaxial applied pressure, as shown in Figures 5(a and b). Pores at the boundary of the SPS Al-CNF compact are mainly created by the chemical etching process, because these were not seen before the etching. The SPS compact shows about $4 \%$ porosity, as indicated in Table I.

Comparing the Al particle size in the SPS compact (Fig. 5) and the original size (Fig. 4), no significant grain growth has occurred after the SPS process. The rapid processing cycle of the SPS process and the pinning effects of the CNFs and $\mathrm{Al}$ oxide act as grain growth inhibitors. In general, the surface of the gas atomized Al particles are surrounded by a few $\mathrm{nm}$ thick $\mathrm{Al}$ oxide layer and this layer also restrains the grain growth. ${ }^{29,30,35,36}$ Most of the interfaces in the SPS Al-CNF compact consist of the Al$\mathrm{O}-\mathrm{CNF}-\mathrm{O}-\mathrm{Al}$ and Al-O-Al systems. However, some contaminants or oxide can be removed by the micro-plasma generated during the SPS process, resulting in the Al-CNF$\mathrm{Al}$ and $\mathrm{Al}-\mathrm{Al}$ interface systems. ${ }^{29,30,37-39} \mathrm{~A}$ similar phenomenon was also demonstrated in the case of Al-CNT composites, as reported previously. ${ }^{29,30}$ 

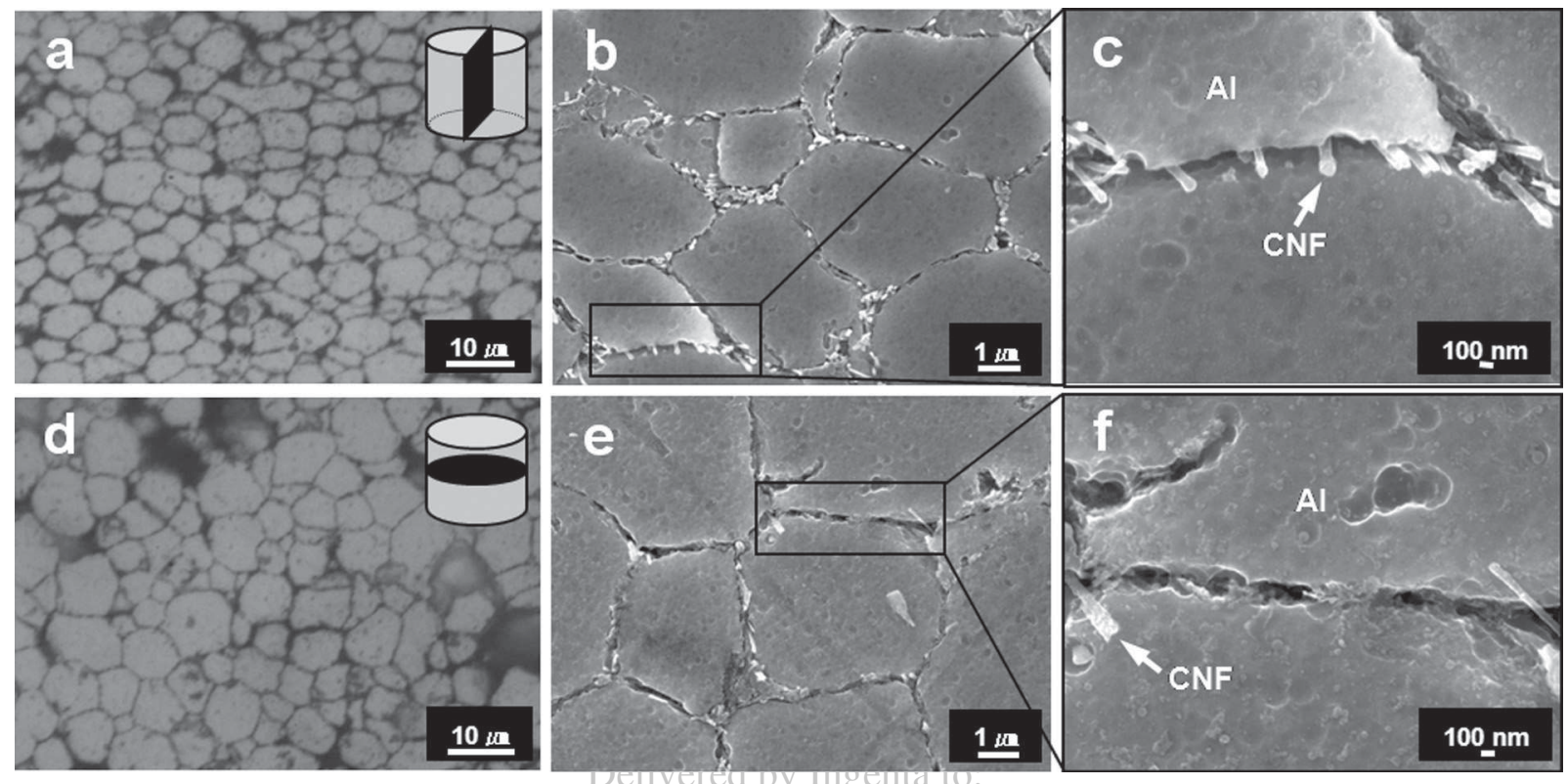

Fig. 5. Micrographs of (a, b, and c) longitudinal and (d, e, and f) transverse cross sections of the SPS Al-CNF compact.

IP : 130.34 .32 .75

Table I. Some properties of the Al and Al-CNF bulk materials. May 2011 06:02:13

\begin{tabular}{lccccc}
\hline Material & $\begin{array}{c}\text { Processing } \\
\text { method }\end{array}$ & $\begin{array}{c}\text { Density } \\
\left(\mathrm{g} / \mathrm{cm}^{3}\right)\end{array}$ & $\begin{array}{c}\text { Tensile strength } \\
(\mathrm{MPa} \pm 2.7, \text { stand. dev. })\end{array}$ & $\begin{array}{c}\text { Elongation } \\
(\% \pm 0.9, \text { stand. dev. })\end{array}$ & $\begin{array}{c}\text { Young's modules } \\
(\mathrm{GPa} \pm 2.8, \text { stand. dev. })\end{array}$ \\
\hline Al bulk & SPS + Extrusion & 2.70 & 52 & 19.5 & 72 \\
Al-CNF bulk & SPS & 2.57 & - & - & - \\
Al-CNF bulk & SPS + Extrusion & 2.62 & 195 & 11.0 & 137 \\
\hline
\end{tabular}

Figure 6 shows micrographs of longitudinal $(\mathrm{a}, \mathrm{b}$, and $\mathrm{c})$ and transverse ( $\mathrm{d}$, e, and $\mathrm{f}$ ) cross sections of the extrudate of the SPS Al-CNF compacts with around $2 \%$ porosity level, as indicated in Table I. The microstructures of the longitudinal and transverse cross sections in the extrudate were totally different, as shown in Figures 6(a and d). The CNFs are highly oriented parallel to the extrusion direction. The many CNF tips observed in the transverse cross

\section{AMEHICAN}

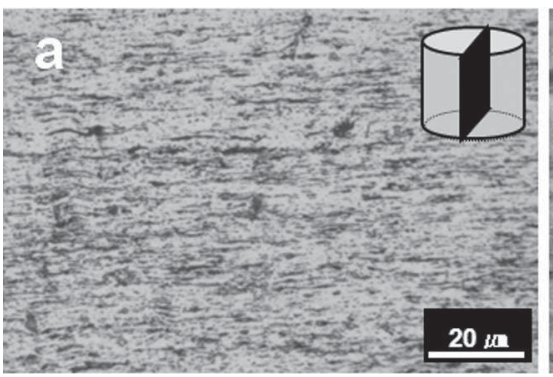

SGITMTIFIC
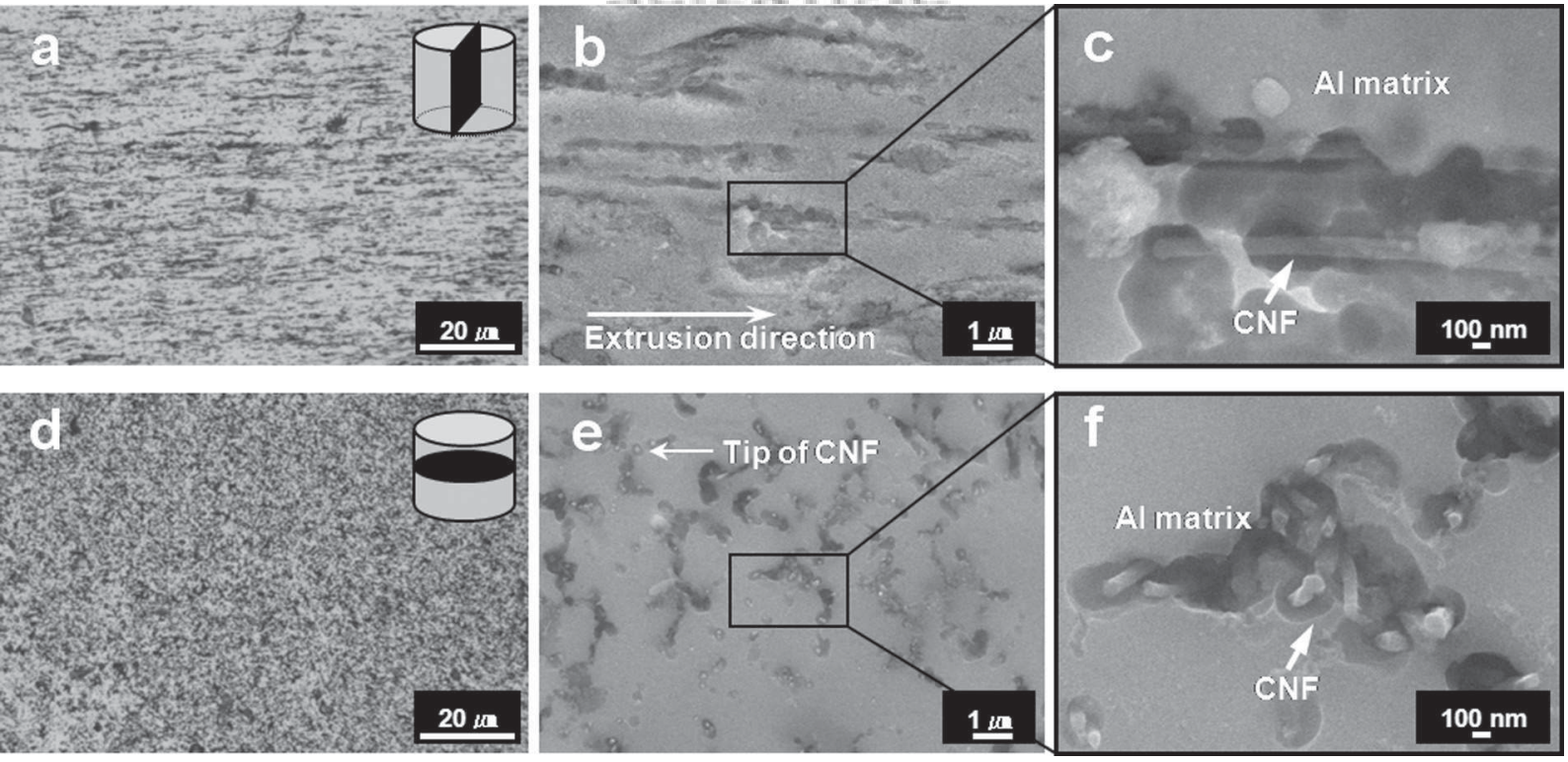

Fig. 6. Micrographs of ( $a, b$, and c) longitudinal and (d, e, and f) transverse cross sections of the extrudate of the SPS Al-CNF compacts. 
section of the extrudate also indicated high directionality of the CNF, as shown by the white arrow in Figure 6(e). The black color in Figures 6(a and d) mainly indicates the interface zone in the Al-CNF extrudate. These uniform microstructures were observed in every part of the sample. According to the high magnification image, the CNF was present in every interface, as shown in Figures 6(b, c, e, and f). In other words, the combination of SPS and hot extrusion processes is able to produce highly oriented and dispersed CNF reinforced Al matrix bulk materials.

Figure 7 shows nominal stress-strain curves for the extruded Al-CNF bulk materials. The pure Al bulk was also fabricated under the same conditions for comparison. The tensile strengths of the extruded Al-CNF bulk materials were three times greater than those of the pure $\mathrm{Al}$ bulk fabricated by the same route, as indicated in Table I. This increase of tensile strength is very likely due to work hardening, as a result of the high plastic deformation process. However, the work hardening hardly affected the enhancement of the tensile strength in the Al-CNF bulk material due to the dynamic recovery that occurred during the extrusion process, because this effect was greater than the accumulation of dislocations. ${ }^{40,41}$ We have compared the hardness of raw $\mathrm{Al}$ particles and an $\mathrm{Al}$ particle in extruded sample by nanoindentation and similar values were obtained. Our previous study also showed a similar result, ${ }^{29}$ i.e., highly enhanced tensile strength of the AlCNF bulk material mainly attributed to the reinforcement produced by $\mathrm{CNF}$ addition.

The measured tensile strength of the Al-CNF bulk materials was compared with the value calculated using the Kelly-Tyson model (ignoring size effects), which is useful for understanding the tensile strength of fiber reinforced materials. $^{43}$

$$
\sigma_{\mathrm{c}}=\left(1-\frac{l_{\mathrm{c}}}{2 l}\right) \sigma_{\mathrm{f}} V_{\mathrm{f}}+\sigma_{\mathrm{m}}\left(1-V_{\mathrm{f}}\right)
$$

where $V_{\mathrm{f}}, \sigma_{\mathrm{f}}$, and $\sigma_{\mathrm{m}}$ represent the volume fraction of fiber, strength of fiber and strength of matrix, respectively. $\sigma_{\mathrm{f}}$ in this study was assumed to be 4-6 GPa. ${ }^{44} V_{\mathrm{f}}$ and $\sigma_{\mathrm{m}}$ are substituted by $5 \mathrm{vol} \%$ and $50 \mathrm{MPa}$. $l_{\mathrm{c}}$ means the length of the fiber when the composite material is broken. It is assumed that $l_{\mathrm{c}}$ equals $l$. The theoretical value of $\sigma_{\mathrm{c}}$ was calculated to be $148-198 \mathrm{MPa}$, as shown in Figure 7. Surprisingly, the experimental value of the tensile strength was located in the theoretically calculated range of values even though an ideal case was assumed, with perfect alignment of homogeneously well dispersed CNFs in the matrix and perfect adhesion between the $\mathrm{Al}$ and CNFs.

We have now attempted to understand how the tensile strength and Young's modulus of Al-CNF bulk materials has become dramatically enhanced over that of pure Al. The high degree of dispersion and alignment of the CNFs are largely responsible for the enhancement of mechanical properties of the Al-CNF bulk materials, as shown in Figure 6. Further microstructural observations using TEM are shown in Figures 8(a and b). The CNFs were shown niversity

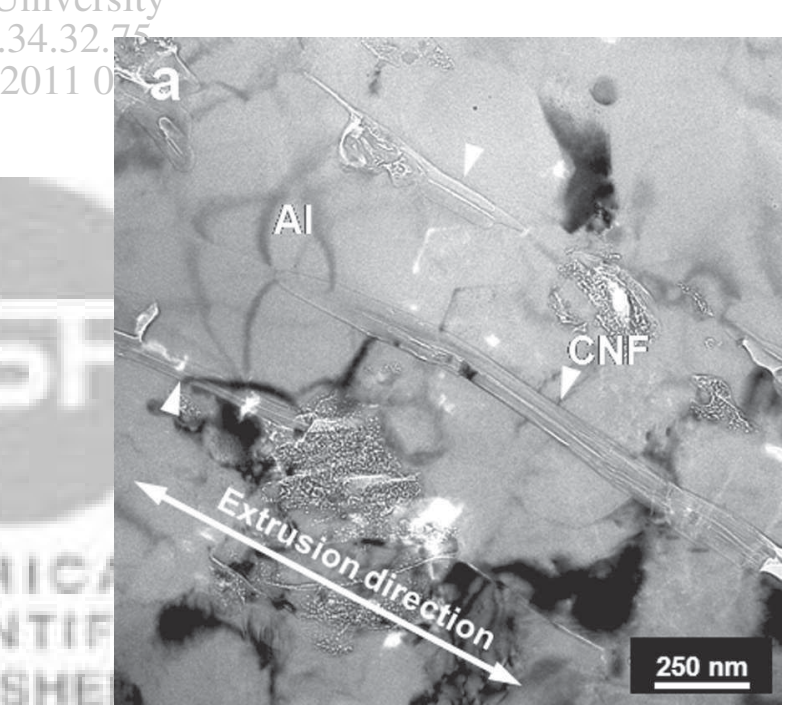

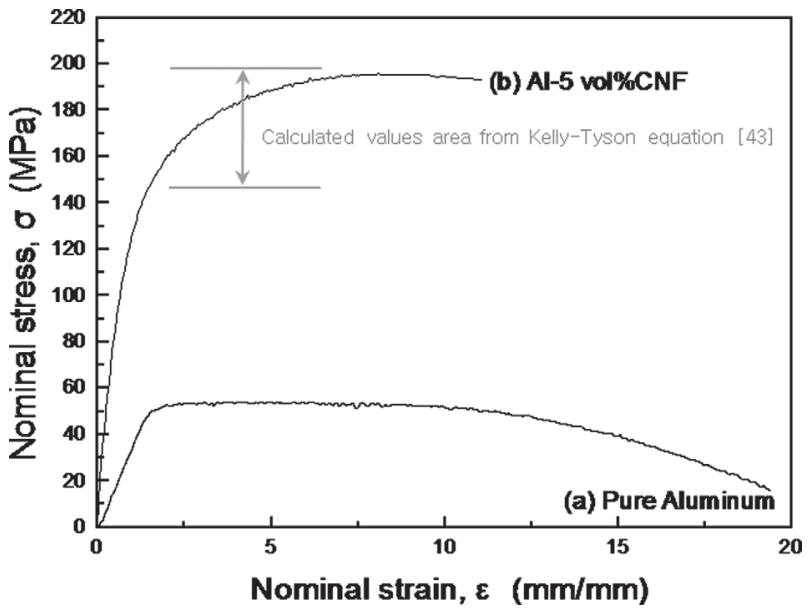

Fig. 7. Nominal stress-strain curve of the extruded pure $\mathrm{Al}$ and extruded Al-CNF bulk materials.

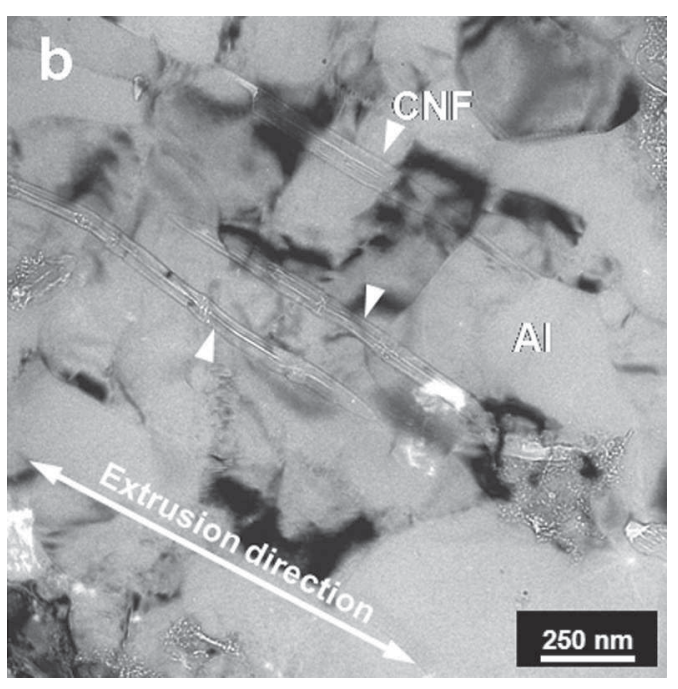

Fig. 8. TEM micrographs of the extruded Al-CNF bulk materials. 

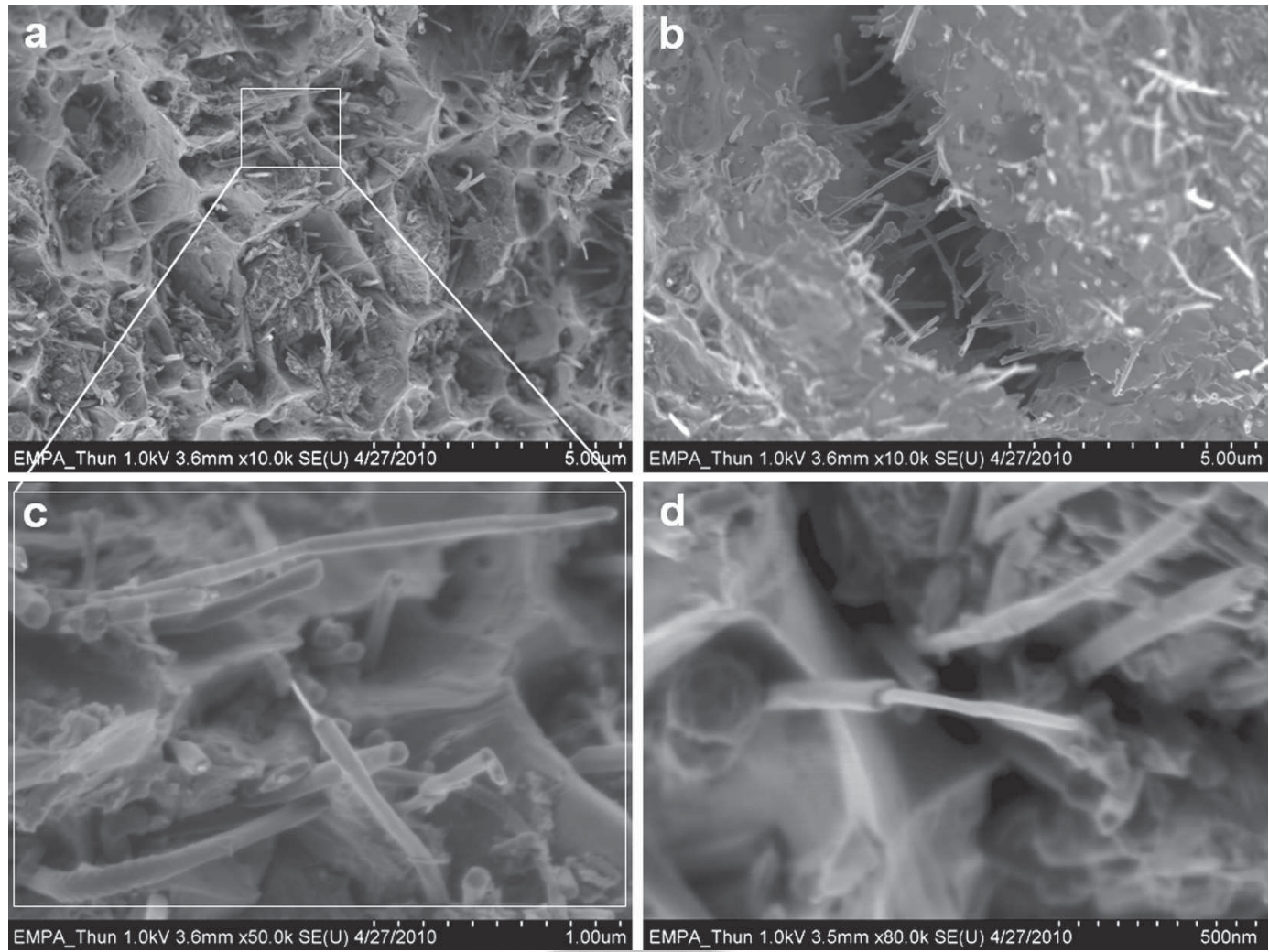

Fig. 9. FE-SEM micrographs of the fracture surface of the extruded Al-CNF bulk materials.

to be very tightly packed and uniformly adhered to the $\mathrm{Al}$ matrix, a microstructure which is useful for load transfer from the $\mathrm{Al}$ matrix to the reinforcement provided by the CNFs. ${ }^{19,43,45-47}$

Many bridging CNFs have been observed and these have also helped to increase the mechanical properties of the Al-CNF bulk material, as shown in Figure 9. These bridgings also contributed to an efficient load transfer from the $\mathrm{Al}$ matrix to the CNF reinforcement under the stress propagation environment. Moreover, the fracture surface of AlCNF bulk material shows a lot of dimples. This implies that the Al-CNF bulk material has basically followed ductile fracture behavior under the tensile stress, resulting in $11 \%$ of elongation, as indicated Table I.

Figure 10 shows $\mathrm{Al}$ carbide $\left(\mathrm{Al}_{4} \mathrm{C}_{3}\right)$ generated at (a) the tip of a CNF and (b) at the bending part of a CNF. The $\mathrm{Al}_{4} \mathrm{C}_{3}$ was generated during the SPS process, which has a high possibility of producing a liquid $\mathrm{Al}$ phase even in the solid state sintering temperature region, ${ }^{29,30,48}$ due to reaction and diffusion between the $\mathrm{Al}$ matrix and the disordered CNFs. ${ }^{1,49,50}$ In general, the $\mathrm{Al}_{4} \mathrm{C}_{3}$ decreases thermal and electrical properties in materials. ${ }^{1}$ However, the presence of $\mathrm{Al}_{4} \mathrm{C}_{3}$ also contributes to improvement of adhesion between the $\mathrm{Al}$ matrix and the carbon based
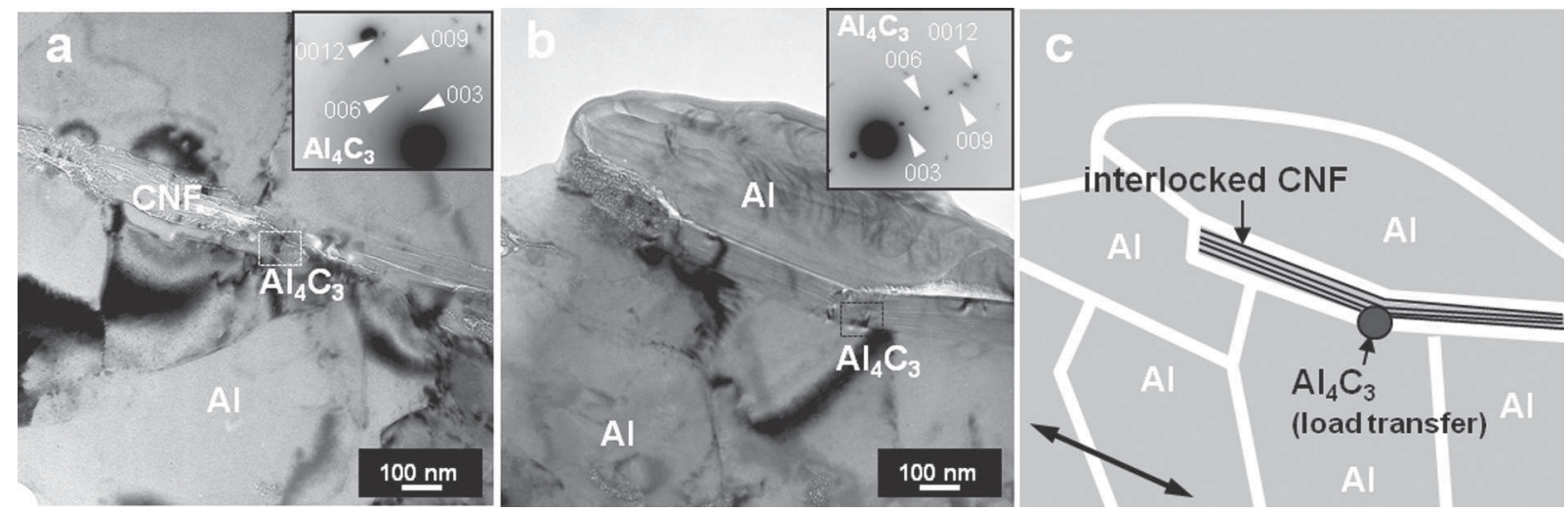

Fig. 10. TEM micrographs of the extruded Al-CNF composites (a and b) and (c) a schematic diagram of (b). 
materials, ${ }^{29,30,49,50}$ i.e., the stress can be transferred efficiently from the $\mathrm{Al}$ matrix to the reinforcing $\mathrm{CNFs}$ via the $\mathrm{Al}_{4} \mathrm{C}_{3}$. Furthermore, different coefficients of thermal expansion (CTE) at the interface between the $\mathrm{Al}$ and $\mathrm{Al}_{4} \mathrm{C}_{3}$ or $\mathrm{CNF}^{15}$ leads to lots of dislocations, which may provide strengthening for the Al-CNF bulk materials.

From a mechanical viewpoint, the $\mathrm{Al}_{4} \mathrm{C}_{3}$ can function as resistance elements in the Al-CNF bulk materials due to their small (nanoscale) size. However, it is necessary to be careful of $\mathrm{Al}_{4} \mathrm{C}_{3}$ formation, because as well as improving adhesion between the $\mathrm{Al}$ matrix and the $\mathrm{CNF}$ it can also demonstrate its natural adverse brittle and hygroscopic properties. ${ }^{1,49,50-52}$ Further details of the effects of $\mathrm{Al}_{4} \mathrm{C}_{3}$ need to be investigated. Finally, the actual shape of the CNF introduced into the Al matrix has also helped to strengthen the Al-CNF bulk materials. Some bent CNFs have been observed, as shown in Figure 10(b). This means that the bent $\mathrm{CNF}$ is biting into the $\mathrm{Al}$ matrix and providing physical bonding between the $\mathrm{Al}$ matrix and the CNF, as shown in Figure 10(c), resulting in an increase of the mechanical properties of the Al-CNF bulk materials. Consequently, the Al-CNF bulk materials are not only enhanced by the addition of CNF but also strengthened by additional several synergistic effects.

\section{CONCLUSIONS}

Homogeneously well dispersed and highly oriented CNF reinforced $\mathrm{Al}$ matrix bulk materials have been successfully fabricated by a combination of spark plasma sintering and hot extrusion. The shape of the CNFs has contributed to high dispersion and directionality of the CNFs in the $\mathrm{Al}$ matrix. The tensile strength of the fabricated Al-CNF bulk materials were enhanced by a factor of $2-3$ over that of the pure Al. This is a result of

(1) The presence of well dispersed and oriented CNFs in the $\mathrm{Al}$ matrix.

(2) a large number of bridging CNFs contributing to efficient load transfer.

(3) efficient transfer of stress from the $\mathrm{Al}$ matrix to the reinforcing CNFs through the $\mathrm{Al}_{4} \mathrm{C}_{3}$ generated in the sintering process.

(4) bent CNFs biting into the Al matrix and providing physically strong bonding between the Al matrix and the CNF.

These results demonstrate that CNFs are a suitable reinforcement material, at least to overcome poor dispersion in the Al matrix bulk materials. We believe that CNFs can also be used for engineering reinforcement of other matrix materials such as ceramics, polymers and complex matrices.

Acknowledgments: This research was supported by the New Energy and Industrial Technology Development Organization (NEDO) of Japan. T. Miyazaki is gratefully acknowledged for help with the HRTEM. We would also like to acknowledge Nissin Kogyo Co. Ltd. for their technical support.

\section{References and Notes}

1. Y. Tang, L. Liu, W. Li, B. Shen, and W. Hu, Appl. Surf. Sci. 255, 4393 (2009).

2. S. J. Park and M. S. Cho, Carbon 38, 1053 (2000).

3. T. Suzuki, Comp. Sci. Technol. 56, 147 (1996).

4. F. F. Komarov and A. M. Mironov, Phys. Chem. Solid State 5, 411 (2004).

5. M. F. Yu, O. Laurie, M. J. Dyer, K. Moloni, T. F. Kelly, and R. S. Ruoff, Science 287, 637 (2000).

6. P. Kim, L. Shi, A. Majumdar, and P. L. McEuen, Phys. Rev. Lett. 87, 2155021 (2001).

7. G. D. Zhan, J. D. Kunts, J. Wan, and A. K. Mukherjee, Nat. Mater. 2, 28 (2003).

8. K. Balani, S. R. Bakshi, Y. Chen, T. Laha, and A. Agarwal, J. Nanosci. Nanotechnol. 7, 3553 (2007)

9. T. Kuzumaki, K. Miyazawa, H. Ichinose, and K. Ito, J. Mater. Res. 9, 2445 (1998)

10. C. F. Deng, D. Z. Wang, X. X. Zhang, and A. B. Li, Mater. Sci. Eng., A 444, 138 (2007).

11. S. R. Bakshi, V. Singh, K. Balani, D. G. McCartney, S. Seal, and A. Agarwa, Surf. Coat. Technol. 202, 5162 (2008).

12. T. Laha and A. Agarwal, Mater. Sci. Eng., A 480, 323 (2008).

13. T. Laha, S. Kuchibhatla, S. Seal, W. Li, and A. Agarwal, Acta Mater. 55, 1059 (2007).

14. T. Laha, A. Agarwal, T. McKechnie, and S. Seal, Mater. Sci. Eng., A 381, 249 (2004).

15. R. George, K. T. Kashyap, R. Rahul, and S. Yamadagni, Scr. Mater. 53, 1159 (2005).

16. A. M. K. Esawi and M. Borady, Compos. Sci. Technol. 68,486 (2008).

17. A. Esawi, K. Morsi, A. Sayed, A. Gawad, and P. Borah, Mater. Sci. Eng., A 508, 167 (2009).

18. K. Morsi, A. ElDesouky, B. Johnson, A. Mar, and S. Lanka, Scr. Mater. 61, 395 (2009).

19. K. Morsi, A. M. K. Esawi, S. Lanka, A. Sayed, and M. Taher, Composites $A 41,322$ (2010).

20. J. P. Salvetat-Delmotte and A. Rubio, Carbon 40, 1729 (2002).

21. C. L. Xu, B. Q. Wei, R. Z. Ma, J. Liang, X. K. Ma, and D. H. Wu, Carbon 37, 855 (1993).

22. A. M. K. Esawi and K. Morsi, Composites A 38, 646 (2007).

23. J. Hilding, E. A. Grulke, Z. G. Zhang, and F. Lockwood, J. Dispers. Sci. Technol. 1, 1 (2003).

24. L. Wang, H. Choi, J. M. Myoung, and W. Lee, Carbon 47, 3427 (2009).

25. http://en.wikipedia.org/wiki/Carbon_nanofiber

26. M. Endo, Y. A. Kim, T. Hayashi, K. Nishimura, T. Matusita, K. Miyashima, and M. S. Dresselhaus, Carbon 39, 1287 (2001).

27. M. Endo, K. Takeuchi, K. Kobori, K. Takahashi, H. W. Kroto, and A. Sarkar, Carbon 33, 873 (1995).

28. M. Endo, Y. A. Kim, T. Takeda, S. H. Hong, T. Matsushita, T. Hayashi, and M. S. Dresselhaus, Carbon 39, 2003 (2001).

29. H. Kwon, M. Estili, K. Takagi, T. Miyazaki, and A. Kawasaki, $\underline{\text { Car- }}$ bon 47, 570 (2009).

30. H. Kwon and A. Kawasaki, J. Nanosci. Nanotechnol. 9, 6542 (2009).

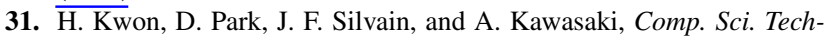
nol. 70, 546 (2010).

32. T. Noguchi, A. Magario, S. Fukazawa, S. Shimizu, J. Beppu, and M. Seki, Mater. Trans. 45, 602 (2004).

33. Q. Zhao and H. D. Wagner, Phil. Trans. R. Soc. A 362, 2407 (2004). 
34. M. S. Dresslhaus, G. D. Dresselhaus, R. Saito, and A. Jorio, $\underline{\text { Physics }}$ Reports 409, 47 (2005).

35. M. Omori, Mater. Sci. Eng., A 287, 183 (2000).

36. G. Xie, O. Ohashi, T. Yoshioka, M. Song, K. Mitsuishi, H. Yasuda, K. Furuya, and T. Noda, Mater. Trans. 42, 1846 (2001).

37. G. Xie, O. Ohashi, K. Chiba, N. Yamaguchi, M. Song, K. Furuya, and T. Noda, Mater. Sci. Eng., A 359, 384 (2003).

38. M. Kubota, J. Alloys Compd. 434, 294 (2007).

39. M. Zadra, F. Casari, L. Girardini, and A. Molinari, Powder Metall. 50, 40 (2007).

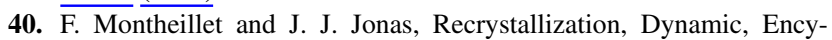
clopedia of Applied Physics, VCH Publishers, Inc. (1996), Vol. 16, pp. 205-225.

41. S. Serajzadeh, Modelling Simul. Mater. Sci. Eng. 12, 1185 (2004).
42. R. Kaibyshev, K. Shipilova, F. Musin, and Y. Motohashi, Mater. Sci. Eng., A 396, 341 (2005).

43. A. Kelly and W. R. Tyson, J. Mech. Phys. Solids 13, 329 (1965).

44. http://www.sdk.co.jp/html/english/index.html

45. K. T. Kim, S. I. Cha, and S. H. Hong, Mater. Sci. Eng., A 430, 27 (2006).

46. H. L. Cox, Br. J. Appl. Phys. 3, 72 (1952).

47. O. Lourie and H. D. Wagner, Comp. Sci. Technol. 59, 975 (1999).

48. T. Nagae and S. T. Yokota, and M. Toyama, Industrial Technology Center 89, 89 (2001).

49. P. W. Ruch, O. Beffort, S. Kleiner, L. Weber, and P. J. Uggowitzer, Comp. Sci. Technol. 66, 2677 (2006).

50. L. Ci, Z. Ryu, N. Y. Jin-Phillipp, and M. Rühle, Acta Mater. 54, 5367 (2006).

51. M. Lancin and C. Marhic, J. Eur. Ceram. Soc. 20, 1493 (2000).

52. W. S. Jung and H. U. Joo, J. Alloys Compd. 465, 265 (2008).

Received: 31 August 2010. Accepted: 2 October 2010. 